\title{
Observações sobre algumas hemogregarinas das aves
}

PELO

\author{
Dr. Henrique de Beaurepaire Aragão \\ Assistente
}

(Com as estampas 2 • 3 )

\section{Beobachtungen über Hämogregarinen von Vögeln VON}

\author{
Dr. Henrique de Beaurepaire Aragão \\ Assistenten am Institut
}

(Hierzu Tafeln 2 und 3)

A existencia de parazitos, semelhantes as hemogregarinas, em animais de sangue quente começou a ser assinalada a partir de 1905 e hoje o numero delles já é bastante elevado. Nestes animais tais protozoarios encontram-se de preferencia no interior dos globulos brancos, donde a denominação impropria de leucocitozoarios que só cabe aos parazitos do tipo do $L$. Ziemanni.

Para evitar essa denominação pouco acertada, outras têm sido propostas, como a de hepatozoon (MILLER I908 e a de leucocytogregarina (PORTER 1909). Preferimos conservar para tais hematozoarios a denominação de hemogregarinas até que conhecimento mais completo do ciclo delles venha determinar a sua colocação definitiva.

Como contribuição para o conhecimento deste grupo particular de hemogregarinas parazitos dos leucocitos, vimos aqui assinalar um certo numero dellas, que observámos desde algum tempo, em aves, nas quais não nos consta terem sido até agora
Das Vorkommen von den Hämogregarinen ähnlichen Parasiten bei warmblütigen Tieren wurde von I905 an bemerkt und heute ist ihre $\mathrm{Zahl}$ schon eine ziemlich grosse. Diese Protozoon zeigen sich bej solchen Tieren besonders im Innern der weisen Blutkörperchen, was zu der unrichtigen Bezeichnung Leukozytozoen geführt hatte, welche nur Paraziten vom Typus des Leukozytozoon Ziemanni zukömmt.

Um diese ungeeignete Bezeichnung zu ersetzen, wurden andere vorgeschlagen, wie Hepatozoon (MILLER I908) und Leucocytogregarina (PORTER I909). Ich ziehe vor einen Blutparasiten den Namen Hämogregarinen beizubehalten, bis eine genauere Kenntnis ihres Entwicklungscyclus ihre definitive Unterbringung gestattet.

Als Beitrag zu dieser eigenartigen Gruppe der leukozytenbewohnenden Hämogregarinen mache ich eine Mitteilung über einige von mir seit einiger Zeit beachtete Arten aus Vögeln, bei denen sie, meines 
encontradas; ha apenas uma lijeira referencia de LAveran (I909) e um trabalho de ADIE (I909), nos quais comtudo esses autores não classificam de hemogregarinas os parazitos observados. Julgando todavia que se trata de hemogregarinas como as nossas, propomos para ellas as denominações de hemogregarina paddae e hemogregarina Adiei.

As aves, que até agora encontrámos infetadas, são as seguintes: a sporophila albogularis SPIX, vulgarmente conhecida por coleiro ou papa capim, a Brachyspiza capensis RIDGw., (tico-tico); a Atticora cyanoleucus, VIEILI; (andorinha) a Sicalis flaveola LiNN., (canario da terra); a Tanagra palmarum WIED.; o Sanhaçú; a Poroaria larvata BoDd., (cardeal); o Rhamphocellus brasilius LINN., (tié sangue.) Seguimos a nomenclatura cientifica das aves, adotada pelo prof. IHERING no seu trabalho: Catalogo da fauna brasileira, Vol. I. Aves. S. Paulo, I907.

As observações que aprezentamos sobre os parazitos destas aves são rezultado de rapida observação e esperamos poder completal-os ulteriormente.

\section{MATERIAL E TECNICA.}

Das aves aqui encontradas infetadas, umas, como os Sporophila, Brachyspiza e as Atticora provem de Manguinhos e arredores; o Rhamphocoelus e a Tanagra foram caçadas no Xerém, E. do Rio; destas recebemos preparações de sangue $e$ esfregaços de pulmão por obsequio dos nossos colegas Drs. NeIva e F ARIA, aos quaes ficamos muito gratos. Recebemos as Poroaria e Sicalis do Estado da Bahia por gentileza de nosso colega Dr. FigueIREdo DE VAsconcellos e do Dr. Guerreiro de Castro.

A pesquiza do parazito deve ser feita tanto no sangue periferico, como nos organs internos, porque nestes não só se encontram muitas vezes parazitos, quando faltam na periferia, como tambem formas de multiplicação do protozoario. Os esfregaços do pulmão são os que revelam com
Wissens noch nicht gefunden wurden. Es giebt darüber eine kurze Mitteilung von Laveran (I900) und eine Arbeit von Adie (1909), in welchen die Autoren die beobachteten Parasiten aber nicht als Hämogregarinen bestimmen. Da ich aber glaube, dass es sich um solche handelt, schlage ich die Namen Hämogregarina paddae und $H$. Adie vor.

Die Vögel, die ich bisher infiziert fand, gehören zu folgenden Arten: Sporophila albogularis SPIX, Brachyspiza capensis RIDGw., Atticora cyanoleucus VIEILL., Sicalis flaveola, Tanagra palmarum, WIEd, Poroaria larvata BodD und Ramphocoelus brasilius L.

Ich folge die Nomenklatur, welche Dr. v. Ihering in seinem a Katalog der brasilianischen Fauna ১ (Bd. I, Vögel S. Paulo 1907) gebraucht. Meine Mitteilung über die Hämogregarinen der angeführten Vögel sind das Resultat einer kurzen Beobachtung, welche ich später zu ergänzen hoffe.

\section{MATERIAL UND TECHNIK.}

Von den infizierten Vögeln stammen die einen, wie Sporophila, Brachyspiza, und Atticora aus Manguinhos und Umgebung, Ramphocoelus und Tanagra dagegen aus Xerém im Staate Rio; letzteren erhielt ich von meinen Kollegen Drs. NeIVA und Faria Blutpräparate und Lungenausstriche, welche ich bestens verdanke. Poroaria und Sicalis erhielt ich lebend aus dem Staate Bahia durch die Güte meines Kollegen Dr. FigueIREdo DE Vasconcellos und des Herrn Dr. GuerReIro de Castro.

Die Parasiten müssen sowohl im peripheren Blute, wie den inneren Organen aufgesucht werden, weil in letzteren nicht nur solche, die im ersteren fehlen, sondern 
maior frequencia e abundancia os parasitos no interior dos leucocytos, ao passo que as formas de multiplicação são mais frequentes no intestino, figado e medula ossea e muito raras no pulmão.

O exame a fresco foi feito entre lamina e laminula ou então em gota pendente com sangue ou suco dos organs do animal infetado, ás vezes adicionado dum pouco de soro do proprio passaro, quando era necessario diluir o material. Não se recomenda neste cazo juntar agua fiziolojica, porquanto esta faz com que os parazitos percam rapidamente a sua mobilidade.

Para a fixação do material em esfregaços, empregamos o sublimado alcool, segundo o metodo de SchaudinN, e muito frequentemente o alcool metilico. No primeiro cazo, as colorações uzadas foram as hematoxilinas de Delafield e HeIdenhain e no segundo o Gremsa. O sublimado alcool tambem foi empregado para a fixação de organs e para córtes que foram córados pelas hematoxilinas acima mencionadas.

\section{MORFOLOJIA E BIOLOJIA GERAIS DAS HEMOGREGARINAS}

Durante a faze adulta as hemogregarinas das aves são parazitos de leucocitos mononucleares. As celulas parazitadas são encontradas com frequencia na circulação periferica, principalmente quando a infeção é abundante. Quando esta é fraca as celulas parazitadas são encontradas quazi só no pulmão da ave, ás vezes ainda na medula ossea e, muito raramente, nos demais organs. Os leucocitos parazitados não aprezentam modificação que possa ser levada á conta da prezença dos protozoarios. As infeções, mesmo as mais intensas, são bem suportadas pelas aves.

Ao passo que as formas adultas das hemogregarinas são encontradas nos leucocitos, os estadios de equizogonia tem logar em celulas epiteliais do intestino, figado, no pulmão e na medula ossea, porém mais raramente nestes dois ultimos organs.

A forma geral dos parazitos adultos auch besonders die Vermehrungsstadien vorfinden.

Die Untersuchung wurde frisch im Deckglaspräparat oder am hängenden Tropfen mit Blut und Organsaft vorgenommen, wobei oft etwas Blutserum desselben Vogels zugesetzt wurde, wenn eine Verdünnung des Materials nötig war. Ein Zusatz von physiologischem Serum empfihlt sich nicht, weil dabei die Parasiten bald ihre Beweglichkeit einbüssen.

Zur Fixierung der Ausstrichpräparate verwende ich Sublimatalkohol nach ScHAUDINN und häufig auch Methylalkohol. Im ersteren Falle diente zur Färbung Hämotoxylinlösungen nach Delafield und HeIDENHAIN, im letzteren die Methode von Giemsa. Sublimatalkohol diente auch zur Fixierung der für Schnitte bestimmten Organe, wobei dann angegebenen Hämatoxylinlösungen verwendet wurden.

\section{ALLGEMEINE MORPHOLOGIE UND BIOLOGIE DER HAMOGREGARINEN.}

Im erwachsenen Zustande sind die Hämogregarinen der Vögel Parasiten der mononukleären Leukozyten. Die befallenen Zellen finden sich häufig in der peripheren Zirkulation, besonders wenn eine starke Infektion vorliegt. Ist sie dagegen gering, so werden parasitenhaltigen Zellen nur ir den Lungen oder manchmal noch im Knochenmark, weit seltener aber in den übrigen Organen gefunden. Die befallenen Leukozyten zeigen keine Veränderungen, welche auf die Gegenwart der Parasiten zurückzuführen wären und selbst die stärksten Infektionen werden von den Vögeln gut ertragen.

Während die erwachsenen Hämogregarinen in Leukozyten gefunden werden, findet die Schizogonie in Epithelialzellen des Darmes, der Leber, der Lunge und des 
varia entre a de vermiculo, de pequeno crecente ou então oval mais ou menos larga. As hemogregarinas têm dimensões diminutas, não excedendo as maiores a $8,2 \mu$ de comprimento.

No interior dos leucocitos tomam diferentes pozições, ora ocupam apenas o protoplasma, mais ou menos aconchegados ao nucleo, chegando mesmo a aderir a elle, ora aparecem superspostas ao nucleo ou mesmo por dentro da trama nuclear. Não raro, nos cazos de intensas infeções, se encontram 6 e mais parazitos em um só leucocito.

Examinadas a fresco as hemogregarinas quer livres, ou no interior dos leucocitos aparecem com aspeto de pequenos corpusculos branco-amarelados constituidos por protoplasma finamente alveolar, tendo no centro um pequeno nucleo veziculozo, geralmente sem membrana vizivel, e com aspeto mais refrinjente. A cromatina do nucleo, aparece ora sob a forma de massas muito regulares e regularmente dispostas, ora com a maior irregularidade de contorno e colocação.

No protoplasma se veem ás vezes vacuolos e outras vezes, uma pequena massa arredondada refrinjente, atraz do nucleo distinta somente na hemogregarina Atticorae e constituida por uma substancia com carateres córantes de plastina. Esta formação é perfeitamente semelhante ao nucleo de plastina, descrito por Prowazek (r908) na Hemogregarina platidactyli Billet. No cazo, porém, das hemogregarinas das aves e como tambem observámos no drepanidium do Leptodactylus ocellatus o nucleo de plastina é unico, ao passo que na hemogregarina observada por ProwaZEK é duplo.

Examinando a fresco os parazitos, muitas vezes se notam alguns movimentos, quer elles se achem livres, quer no interior dos globulos brancos. No interior destes, os movimentos das hemogregarinas limitam-se a pequenos deslocamentos um pouco bruscos, seguidos de curtos intervallos de repouzo, graças aos quais o parazito consegue libertar-se da celula, no interior da qual se acha. Diferentes fazes da saida
Knochenmarkes statt jedoch seltener in den beiden letzteren Organen.

Die allgemeine Form der erwachsenen Parasiten wechselt zwischen der eines Würmchens eines Halbmondes und eines schmäleren oder breiteren Ovales. Sie haben kleine Dimensionen und selbst die grössten überschreiten die Länge von $8,2 \mu$ nicht.

Im Innern der Leukozyten nehmen sie verschiedene Lagen ein, bald finden sie sich nur im Plasma, dem Kerne mehr oder weniger angeschmiegt, bald scheinen sie auf demselben oder sogar im Innern des Kerngerüstes zu liegen. In Fällen intensiver Infektion finden sich nicht selten 6 oder mehr Parasiten in einer weissen Blutzelle.

Frisch untersucht erscheinen die freien oder in Leukozyten eingeschlossenen Hämogregarinen als kleine gelblichweisse Körperchen, welche aus einem feinwabigen Protoplasma bestehen; in der Mitte liegt ein kleiner, bläschenartiger Kern gewöhnlich ohne deutliche Membran, der stärker lichtbrechend aussieht. Sein Chromatin hat bald die Form sehr regelmässig geformter und angeordneter Klumpen, bald ist es in Umriss und Anordnung höchst irregulär.

Im Protoplasma sieht man manchmal Vakuolen und manchmal hinter dem Kerne ein rundliches lichtbrechendes Körperchen, das nur bei Hämogregarina atticorae deutlich und aus einer, wie Plastin färbbaren Substanz besteht. Diese Bildung gleicht völlig dem von v. Prowazek bei Hämogregarina platydactyli BILLET beschriebenen Plastinkerne. Jedoch 'ist derselbe bei den Hämogregarinen der Vögel, wie ich auch beim Drepanidium von Leptodactylus ocellatus beobachtete in der Einzahl vorhanden, während er bei der von $v$. Prowazek beobachteten Hämogregarine doppelt ist.

Beobachtete man lebende Parasiten frei oder im Innern von Leukozyten, da beobachtete man nicht selten einige Bewegungen. Bei den eingeschlossenen beschränken sie sich auf kleine, ziemlich plötzliche Bewegungen, welche von kurzen 
dum parazito do globulo branco vem reprezentadas nas figuras I a 12 da est. I.

Uma vez libertadas do leucocito que parazitavam, as hemogregarinas exibem movimentos mais ativos e mais amplos. Vê-se então o parazito contrair-se para o lado da sua concavidade repetidas vezes e depois bruscamente distender-se, retomando a primitiva forma; graças á rapidez com que executa este movimento, se desloca na preparação, descrevendo pequenos trajetos em arco. Geralmente, na execução destes movimentos, o prazito conserva imobilizada a porção posterior e só a anterior se contrae. Além dos movimentos acima descritos, que são os mais amplos, os parazitos executam ainda pequenas contrações e contorsões sem abandonar quazi a pozição, em que se acham na preparação. Os movimentos das hemogregarinas se repetem por cerca de 3 a 4 horas, com intensidade decrecente, e findo este prazo, dezaparecem de todo mesmo que se observem nas melhores condições de temperatura e de meio de vida.

As preparações córadas revelam, entre os parazitos das diferentes aves, grande semelhança de forma e estrutura, porém, com pequenas variações de uma especie para outra. Córadas pelo GIEMSA, as hemogregarinas das aves aprezentam em geral o protoplasma tinto em azul claro, vendo-se nelle, não raro, numerozas granulações vermelhas de substancias de rezerva. O nucleo aprezenta-se formado por massas de cromatina que se córam em vermelho intenso e aprezentam-se ás vezes regularmente dispostas, porém, na regra na maior irregularidade. A existencia de cariozoma e de membrana no nucleo foi distintamente observada apenas na Hemogregarina atticorae (figs. 13,28 est. $\mathrm{r}$ ).

Sobre o dezenvolvimento dos parazitos no organismo das aves o que se observa, examinando animais em periodicos diversos da infeção, é que, no inicio desta, ocorre uma multiplicação rapida do parazito no interior de celulas do intestino, figado, pulmão e medula ossea, seguindose a ella a penetração das hemagregarinas
Ruhepausen gefolgt sind und es dem Para siten möglich machen, sich aus der umschliesenden Zelle $\mathrm{zu}$ befreien In Fig. I-I2, Tafel I sind verschiedene Phasen des Austrittes eines Parasiten aus einem Leukozyten dargestellt.

Einmal aus der bewohnten Blutzelle freigeworden, zeigen die Hämogregarinen lebhaftere und ausgiebigere Bewegungen. Man sieht dann, wie der Parasit sich wiederholt im Sinne der Konkavität zusammenzieht und dann plötzlich bis zur ursprünglichen Form wieder ausdehnt ; in Folge der Geschwindigkeit, mit welcher diese Bewegungen ausgeführt werden, bewegt er sich im Präparate unter Zurücklegung bogenförmiger Strecken. Bei diesen Bewegungen bleibt gewöhnlich der hintere Teil des Parasiten unbeweglich und nur die vordere Partie kontrahiert sich. Ausser den oben beschriebenen stärksten Bewegungen führen die Parasiten noch kleine Kontraktionen und Torsionen aus, ohne ihren Ort im Präparate zu verlassen. Die Bewegungen wiederholen sich in abnehmender Intensität während 3 bis 4 Stunden und verschwinden nach dieser Frist gänzlich, wenn sich auch die Parasiten den günstigsten Temperaturund Lebensbedingungen befinden.

Gefärbte Präparate zeigen bei den Parasiten der verschiedenen Vögel grosse Aehnlichkeit in Form und Struktur, jedoch mit kleinen Abänderungen von einer Art zur anderen. Nach GiEMSA gefärbt zeigen die Hämogregarinen der Vögel ihr Protoplasma von hellem Blau nicht selten mit zahlreichen roten Körnchen von Chromatinmassen $z u$ bestehen, welche sich intensiv rot färben; manchmal sind sie gleichförmig umgeordnet, gewöhnlich jedoch höchst unregelmässig. Das Vorkommen von $\mathrm{Ka}$ riozom und Membran wurde nur beim Kerne von Haemogregarina atticorae deutlich erkannt (Fig. 13, 29, Tafel I).

Wenn man Vögel in verschiedenen Infektionsperioden untersucht, so beobachtet man in Bezug auf die Entwiklung der Parasiten in ihrem Organismus, dass eine rasche Vermehrung in den Zellen des 
nos leucocitos e a sua passajem para a circulação.

Esta esquizogonia inicial foi por nós observada em quazi todas as suas fazes na Hemogregarina sporophylae (figs. $3^{8}$ a 46 , est. I e 47 a 50 e 59 est. II), e alguns dos estadios na Hemogregarina Sicalidis (fig. 72, est. II) e na Hemogregarina poroariae (fig. 76, est. II). Até agora não conseguimos surpreender, qual a forma inicial $\mathrm{da}$ infeção da ave. A julgar pelas verificações, feitas por MILler no Hepatozoon perniciosum, é de prever que seja um produto da evolução esporogonica do parazito em um hospedeiro intermediario. Os elementos que se vão multiplicar por esquizogonia penetram no protoplasma da celula epitelial, aumentam rapidamente de volume, tomam a forma arredondada $e$ entram em rapida multiplicação nuclear. O protoplasma da celula é rapidamente invadido pelo parazito, hipertrofia-se e o nucleo é recalcado. A membrana, que a principio limita o parazito, dezaparece e este se expande por todo o protoplasma da celula sob a forma de uma grande massa de cromatina com abundantes e irregulares corpusculos. Finalmente a esquizogonia se termina pela fragmentação da massa protoplasmica em numerozissimos merozoitos pequenos com forma de virgula larga. Estes são constituidos por um protoplasma, sem vacuolos, nem grandes alveolos, e aprezentam no interior o nucleo sob a forma de duas pequenas massas de cromatina, ligadas entre si por pequena faixa de substancia acromatica. Não raro se vê, junto ao nucleo, uma pequena porção de substancia plastinica (nucleo de plastina ?) especialmente distinta nos preparados córados pelo Gremsa (figs. 38-4I. est I e 59, est. II).

Estes pequenos merozoitos penetram nos leucocitos, onde a principio conservam o seu aspeto carateristico (figs. 52, 53. $74 \mathrm{e}$ 75 , est. II) ; aos poucos, porem, aumentam de volume e adquirem a morphologia que descrevemos para as formas adultas.

No organismo das aves não conseguimos obșervar outra modalidade de dezen-
Darmes, der Leber, der Lunge und des Knochenmarks stattfindet, auf welche das Eindringen der Hämogregarinen in die Leukozyten und ihr Uebertritt in die Zirkulation folgt.

Diese anfängliche Schizogonie wurde von mir in beinahe sämmtlichen Phasen der H. sporophilae (Fig. 38-46, Taf. I, $47-50$ \& 59, Taf. II) beobachtet, dagegen nur in einigen derselben bei $H$. sicalidis (Fig. 72, Taf. II) und $H$. poroariae (Fig. 76, Taf. II). Bis jetzt gelang es nicht, die Form aufzudecken, welche bei den Vögeln die Infektion einleitet. Nach den von MILler bei Hepatozoon perniciosum gemachten Erhebungen kann man erwarten, dass es sich um das Produkt einer Sporogonie des Parasiten in einem $\mathrm{Zwi}$ schenwirt handelt. Die Elemente, welche sich zur Schizogonie vorbereitet, dringen in das Protoplasma der Epithelzelle, vergrössern sich rasch, runden sich $a b$ und zeigen eine schnelle Kernvermehrung. Das Protoplasma der Zelle wird von dem Parasiten bald invadiert, und hypertrophiert, während der Kern bei Seite geschoben wird. Die Membran, welche anfangs den Parasiten abgrenzt, verschwindet und derselbe breitet sich im ganzen Zellprotoplasma aus, in Form eines grossen Klumpens mit zahlreichen Chromatinkörperchen von unregelmässiger Form. Endlich kommt die Schizogonie zum Abschluss, indem das Protoplasma in sehr zahlreiche kleine Merozoiten von breiter Kommaform zerfällt. Diese bestehen aus einem Plasma ohne Vakuolen oder grössere Alveolin und enthalten einen Kern in Form zweier kleiner Chromatinmassen, die unter sich, durch einen Streifen achromatischer Substanz verbunden sind. Nicht selten sieht man nahe am Kern einen kleinen Teil von Plastinsubstanz (Plastinkern), der in nach Giemsa gefärbten Präparaten deutlich ist (Taf. I, Fig. 38-4I, Taf. II, Fig. 59). Diese kleinen Merozoiten dringen in die Leucozyten ein, wobei sie anfangs ihr charakteristisches Aussehen beibehalten (Taf. II, Fig. 52, 53, 74 \& 75); nach und nach vergrössern sie sich aber, wobei sie 
volvimento dos parazitos, além da que acabamos de referir. Tambem não conseguimos, até aqui, observar nas hemogregarinas das aves, diferenças morfolojicas ou córantes que autorizem a distinção de sexualidade.

Foram até aqui negativas as nossas pesquizas para obter a infeção de aves da mesma especie e de especies diversas, quer por inoculação por variadas vias, quer por injestão de material de diferentes organs de aves infetadas.

Igualmente não foram, até aqui, coroadas de sucesso as tentativas, para obter a transmissão dos parazitos dum animal a outro, por meio dum hospedeiro intermediario.

Tendo encontrado numerozos dermanyssus em ninhos de andorinhas, fizemos diversas tentativas de transmissão tanto da Haemogregarina atticorae, como com a Haemogregarina sicalidis. Apenas observámos que os dermanyssus, que sugavam as aves infetadas, aprezentavam até 8 dias depois, no aparelho dijestivo, hemogregarinas ainda moveis; não nos foi, porem, dado observar qualquer evolução dellas nem fenomenos de fecundação. Com mosquitos não colhemos tambem até agora, rezultado algum favoravel.

Passamos a dar pequenas descrições das especies de hemogregarinas, que observámos nas aves, e que, embora guardando entre si estreito parentesco, são, ao nosso ver, especificamente diferentes. As especies, que aprezentamos neste trabalho são as seguintes :

I. H. atticorae; 2. H. ramphoceli; 3. H. poroariae; 4. H. sporophilae; 5. $\mathrm{H}$. tanagrae; 6. H. sicalisis; $7 . \mathrm{H}$. brachyspizae.

Hemogregarina atticorae, n. sp.

(Figs. I3 a 28. Est. I)

Esta especie é encontrada na atticora cyanoleucus, VIEILL, vulgarmente denominada andorinha. Os exemplares infetados, em numero de 3 entre muitos examinados, foram apanhados em Manguinhos. Para- die für den erwachsenen Parasiten bestimmte Form annehmen.

Es gelang mir nicht im Körper der Vögel eine andere Entwicklungsweise, als die eben beschriebene zu beobachten. Ebensowenig gelang es mir, bei den Hämogregarinen der Vögel in Form und Färbung Unterschiede zu beobachten, welche als Geschlechtsdifferenzen gelten könnten.

Meine Uebertragungsversuche bei Vögeln derselben oder verschiedener Arten sowohl durch Einimpfung auf verschiedenen Wegen, als auch Fütterung mit infizierten Organen haben bisher nur negative Resultate ergeben. Ebenso erfolglos waren bisher die Versuche, den Parasiten durch einen $Z$ wischenwirt von einem Vogel auf den anderen $z u$ ubertragen.

$\mathrm{Da}$ ich in einem Schwalbenneste zahlreiche Dermanyssus gefunden hatte, machte ich verschiedene Uebertragungsversuche mit Haemogregarina atticorae und $H$. sicalidis. Ich beobachtete nur, dass die Exemplare des Dermanyssus, welche an infizierten Vögeln gesaugt hatten, noch acht Tagen nachher in Verdauungsapparat bewegliche Hämogregarinen enthielten; doch konnte ich weder eine Weiterentwicklung, noch Fekundationserscheinungen beobachten. Ebenso ungünstig sind die bisher mit Mücken erzielten Resultate.

Ich gebe nun kurze Beschreibungen der von mir bei Vögeln beobachteten Arten, welche, meiner Ansicht nach, obwohl nahe verwandt, doch spezifisch verschieden sind. In dieser Studie führe ich nachfolgende Arten an: I. H. atticorae; 2. H. Ramphocoeli; 3. H. poroariae; 4. H. sporophilae; 5. H. tanagrae; 6. H. sicolidis; 7. H. brachyspizae.

\section{Haemogregarina atticorae n. $\mathrm{s}$.}

(Tafel I, Fig. 13-28)

Diese Art wird bei einer gemeinen Schwalbe, Atticora cyanoleucus VIEILL. gefunden. Die 3 infizierten Exemplare wurden in Manguinhos erbeutet

Parasit leichtgekrümmt, keulen- oder 
zito em forma de clava, lijeiramente encurvada ou de vermiculo, medindo $6,5 \mu$ de comprimento por $2,9 \mu$ de largura. Protoplasma finamente alveolar, córando-se em azul palido pelo Giemsa.

Nucleo, colocado adiante da metade do comprimento do corpo e constituido por um pequeno cariozoma central, quazi sempre unico; este se liga por finos filamentos acromaticos a numerozas pequenas massas de cromatina, situadas na periferia da zona nuclear, junto a delgada membrana, que limita o nucleo externamente. Atraz do nucleo e colada a elle se vê sempre, muito distintamente, uma pequena massa arredondada, que se córa em azul esverdeado pelo Giemsa, o chamado nucleo de plastina. No leucocito parazitado a hemogregarina é encontrada no protoplasma, deitada sobre o nucleo ou mesmo colocada um pouco por dentro duma delgada camada de trama nuclear.

Hemogregarina rhamphocoeli, n. sp.

(Figs. 29 a 34. Est. I)

Esta hemogregarina é parazito do Rhamphocellus brasilius LINN., cuja denominação popular é tié sangue. Os exemplares infetados foram encontrados no Xerém, E. do Rio.

A forma do parazito varia entre a de clava, de vermiculo irregular ou de elipse; as dimensões ocilam entre 8,2 $\mu$ de comprimento por $3,3 \mu$ de largura. Protoplasma finamente alveolar. e córando-se em azul claro, pelo Giemsa. O nucleo é muito irregular e aprezenta-se constituido por pequenas massas de cromatina, ligadas entre si por porções acromaticas. A zona nuclear ocupa um certo espaço, sem limites distintos do protoplasma do parazito. Algumas massas de cromatina se vêm completamente izoladas no protoplasma.

Hemogregarina poroariae, n. sp.

(Figs. 35. a 37. Est. I)

E' parazito da Poroaria larzata BODD., vulgarmente conhecido pelo nome vurmförmig von $6,5 \mu$ Länge und $2,8 \mu$ Breite. Protoplasma feinwabig, bei Giems Afärbung hellblau. Kern vor der Körpermittle fast immer nur mit einem kleinen zentralen Karyosom, welches durch feine achromatische Fäden mit zahlreichen Chrotinklümpchen verbunden ist; diese liegen der Peripherie der Kernsaftzone dicht an der feinen Membran, welche den Kern nach aussen begrenzt. Hinter dem Kerne und mit ihm verklebt sieht man immer sehr deutlich ein rundliches Körperchen, das sich nach Gremsa grünlich brau färbt, den sogenannten Plastinkern. Die Hämogregarine wird im Protoplasma des L.eukozyten gefunden, auf dem Kerne oder selbst unter einer dünnen Schicht des Kerngerüstes gelegen.

\section{Haemogregarina rhamphocoeli n. sp.}

(Tafel I, Fig. 29 34)

Diese Hämogregarine ist ein Parasit des Ramphocoelus brasilius L. Die infizierten Exemplare wurden in Xerém, im Staate Rio gefunden.

Die Gestalt des Parasiten variiert zwischen der Form einer Keule, eines Würmchens oder einer Elipse; die Länge schwankt zwischene 8,2 $\mu$ lang bei einer Breite von $3,3 \mu$. Protoplasma feinwabig, nach Giems a hellblau gefärbt. Der sehr unregelmässige Kern besteht aus Chromatinklümpchen, welche durch achromatische Substanz verbunden sind. Die Kernsaftzone zeigt eine gewisse Breite, ist aber von dem Protoplasma des Parasiten nicht deutlich abgegrenzt. In letzterem sieht man einige ganz isolierte Chromatinmassen.

\section{Haemogregarina poroariae n. sp.}

(Taf. 1, Fig. 35.37)

Parasit der hier als Cardinal bekannten Poroaria larvata BoDd. 
de cardeal. Todos os numerozos exemplares examinados estavam infetados; provinham dos Estados da Bahia e de Minas.

Parazito de forma oval, quazi regular, medindo $5,8 \mu$ de comprimento por $3,3 \mu$ de largura.

Protoplasma finamente alveolar córando-se em azul pelo Giemsa. Nucleo irregular, constituido por diversas massas de cromatina, um pouco para traz da metade do comprimento do parazito. Parazito colocado no protoplasma do leucocito. Foram observadas formas de esquizogonia na medula ossea (fig. 76, est. II).

Hemogregarina sporophilae, n. sp.

(Figs. $3^{8}$ a 46. Est. I, e figs. 47 a 5o. Est. II)

E' encontrada na Sporophilae albogularis SPIX, cujo nome vulgar é coleiro ou papa capim.

Quazi todos os exemplares que se examinam no Rio aprezentam-se infetados.

Parazitos pequenos em forma de clava, virgula, ou de oval, ás vezes, bastante largo, medindo $6 \mu$ de comprimento por $3 \mu$ de largura.

Protoplasma finamente alveolar, córando-se em azul claro pelo Giemsa e ás vezes provido de numerozos granulos vermelhos de substancias de reserva. Nucleo dos parazitos adultos de forma muito irregular, sem contornos nitidos, constituidos por massas de cromatina, mais ou menos numerozas. Os parazitos se encontram no protoplasma dos leucocitos. Nas formas jovens o nucleo é compato. A esquizogonia deste parazito tem logar no figado e no intestino.

\section{Hemogregarina tanagrae}

(Figs. 60 a 63. Est. II)

Parazito da Tanagra palmarum WIED., vulgarmente chamado sanhaçú. As aves, em que foram encontrados os parazitos, foram caçadas no Xerém, E. do Rio.

Parazito, em forma de elipse estreita ou lijeiramente sigmoide medindo $6,3 \mu$ de comprimento por $3 \mu$ de largura. Proto-
Die zahlreiche untersuchten Exemplare aus den Staaten BaHIA und Minas waren sämmtlich infiziert.

Kleine Formen von der Gestalt einer Keule oder eines Kommas oder eiformig; $6 \mu$ lang bei einer Breite von $3 \mu$.

Protoplasma feinwabig, nach Giemsa hellblau gefärbt. Unregelmässiger, aus verschiedenen Chromatirklumpen bestehender Kern, etwas hinter der Mitte des Parasiten gelegen. Parasit im Protoplasma der Leukozyten Schizogonieformen (Taf. II, Fig. 76) wurden in Knochenmark gefunden.

Haemogregarina sporophilae $\mathrm{n}$. $\mathrm{sp}$.

(Taf. I, Fig. 38-46, Taf. II, Fig. 47-58)

Parasit der Sporophila albogularis SpIx. Fast alle in Rio untersuchten Exemplare erwiesen sich infiziert.

Kleine Formen von der Gestalt einer Keule oder eines Kommas oder eiförmig und manchmal ziemlich breit. I.äng $6 \mu$ bei einer Breite von $3 \mu$.

Feinwabiges, nach Giemsa hellblau gefärbtes Protoplasma, oft mit zahlreichen roten Körnern von Reservesubstanzen. Kern der erwachsenen Parasiten sehr unregelmässig und nicht scharf begrenzt, von einer wechselnden Anzahl von Chromatinmassen gebildet. Bei jungen Formen ist der Kern kompakt. Die Parasiten befinden sich im Protoplasma der Leukozyten; die Sporogonie findet in Darm und Leber statt.

\section{Haemogregarina tanagrae}

(Taf. II, Fig. 60-63)

Parasiten von Tanagra palmarum WIED. Die infizierten Vögel wurden in Xerém, im Staate Rio erbeutet.

Körperform schmal elliptisch oder leicht sigmoid $6,3 \mu$ lang und $3 \mu$ breit. Protoplasma wabig, manchmal mit Reser- 
plasma alveolar, ás vezes provido de substancias de rezerva. Nucleo de forma irregular, constituido por massas diversas de cromatina, reunidas entre si. Parazitos colocados no protoplasma dos leucocitos.

Hemogregarina sicalidis, n. sp.

(Figs. 58, 64 e 74. Est. II)

Parazito da sicalis flaveola LINN., vulgarmente conhecido por canario da terra. As aves, infetadas em grande numero provinham do Estado da Bahia.

Parazito em forma de oval irregular, de clava ou de crecente, medindo $5,5 \mu$ por $3,2 \mu$ e menos ainda.

Protoplasma alveolar, córando-se pelo Giemsa em azul e ás vezes com tons rozeos, devido a abundantes de substancias de rezerva. Nucleo mal definido. constituido por massas irregulares de cromatina, ora agrupadas, ora quazi completamente izoladas. A's vezes, se encontram muitos parazitos no mesmo leucocito e neste cazo ocupam tanto o protoplasma, como o nucleo. Foram observadas formas de esquizogonia no pulmão do passaro (fig. 72, est. II).

\section{Hemogregarlna brachyspizae, n. sp.}

(Figs. 75 a 79. Est. II)

Parazito da Brachyspiza capensis RIDG., vulgarmente chamado tico-tico.

Todos os individuos apanhados em Manginhos são infetados. A hemogregarina se aprezenta em geral em forma de oval eu elipse irregulares e mede $6 \mu$ de comprimento por $3 \mu$ de largura.

Protoplasma é finamente alveolar, que se córa em azul pelo Giemsa e não raro aprezenta-se carregado de substancias de reserva. Nucleo muito irregular e constituido por massas de cromatina ligadas entre si. Formas jovens com nucleo compato. O parazito é encontrado no protoplasma dos leucocitos,

Manguinhos, Março de IgIx. vesubstanzen. Unregelmässig geformter Kern, aus verschiedenen, unter sich verbundenen Chromatinmassen bestehend. Parasiten im Protoplasma der Leukozyten.

\section{Haemogregarina sicalidis}

(Taf. II, Fig. 58, $64 \&$ 74).

Parasit von Sicalis flaveola L. als einheimischer Kanarienvogel bekannt. Die grossenteils infizierten Vögel stammten aus dem Staate Bahia.

Gestalt unregelmässig eiförmig oder in der Form einer Keule oder eines Halbmondes $5,5 \mu$ lang und höchstens $3,2 \mu$ breit.

Das wabige Protoplasma färbt sich nach Giemsa blau, und manchmal wegen der reichlich vorhandenen Reservesubstanzen in rõtlichen Tönen. Kern schlecht umschrieben, aus vereizelten oder gruppenbildenden, unregelmässigen Chromatinsubstanzen gebildet. Manchmal finden sich viele Parasiten im selben Leukozyten und nehmen dann sowohl das Protoplasma, wie den Kern ein. Schizogonieformen wurden in den Lungen der Vögel gefunden (Taf. II, Fig. 72).

\section{Haemogregarina brachyspizae}

(Taf. II, Fig. 75-79)

Parasiten von Brachyspiza capensis RIDG., hier als Tico-tico bekannt. Alle in Manguinhos gefangenen Exemplare waren infiziert. Körper unregelmässig eiförmig oder eliptisch $6 \mu$ lang und $3 \mu$ breit.

Das feinwabige Protoplasma färbt sich nach GIEMSA blau und ist nicht selten mit Reservesubstanzen beladen. Der unregelmässige Kern aus miteinander verbundenen Chromatinmassen bestehend. Jüngere Formen mit kompaktem Kerne. Parasit im Protoplasma der Leukozyten.

Manguinhos, März rgrr. 


\section{EXPLICAÇÃO DAS ESTAMPAS}

\section{ESTAMPA I.}

Figs. I a 12. Diferentes fazes da saida duma haemogregarina (h. sporophilae) dum leucocito. Zeiss Comp. ocular compens. 6, Object apochromat. $2 \mathrm{~mm}$.

Figs. 13 a 46. Dezenhadas com a camara clara de Abbé e com o sistema ocul. compensad. 12 onject apochromat. $2 \mathrm{~mm}$. Coloração pelo Gremsa de todos os parazitos salvo os das figs. $3^{8}$ a 41 para as quais foi utilizado a hematox. de Heidenhain.

Figs. 13 a 28. Haemogregarina atticorae.

Figs. 29 a 34 .

Figs. 35 a 37 .

Figs. $3^{8}$ a 46.

$\begin{array}{cl} & \text { rhamphocoeli. } \\ \text { poroariae. } & \\ \text { quizogonia. } & \text { sporophilae es- }\end{array}$

ESTAMPA II.

Figs. 47 a 79 . Dezenhadas da mesma forma que as figs. I3 a $46 \mathrm{da}$ pajina precedente. Todos os preparados foram córados pelo GiEMsA salvo o n. 70 em que a coloração empregada foi a hematox. de Hempentrain.

Figs. 47 a 50. Haemogregarina sporophilae; esquizogonia.

Figs. 51 a $5^{8}$. Haemogregarina sporophilae; parazitos jovens e adultos no interior dos leucocitos.

Fig. 59. Jovens parazitos provenientes duma esquizogonia.

Figs. 60 a 63 . Haemogregarina tanagrae.

Figs. 64 a $71 \mathrm{e}$

Fig. 72 .

73,74 .

Figs 74, 75,

$\begin{array}{cc}77 \text { a } 79 . & \text { Haemogregarina brachyspizae. } \\ \text { Fig. } 76 . & \text { Haemogregarina brachyspizae; }\end{array}$

Haemogregarina sicadilis.

Haemogregarina sicadilis; forma esquizogonica. forma esquizogonica.

\section{ERKLARUNG DER TAFFLN.}

TAFEL I.

Fig. 1-12. Verschiedene Phasen des Austrittes der sporophilae aus einem Leukozyten. Zeiss Comp. oc. 6, Apochr. $2 \mathrm{~mm}$.

Fig. 13-46. Mit Comp. oc. 12, Apochr. 2 mm. und Abbéschem Zeichnungs. apparat aufgenommen. Alle Fig. nach Giemsa, nur $38 \& 4$ i mit Hämatoxylin nach HeIUENHAIN gefärbt.

Fig. 13-28. Haemogregarina atticorae.

Fig. 29-34. D thamphocoeli.

Fig. 35-37.. poroariae.

Fig. $3^{8-46 . ~} \quad$ sporophilae, Schi-

zogonie.

TAFEL II.

Fig. 47-79. Aufnahme, wie bei Fig. I3-46 auf Tafel I. Färbung nach Gremsa nur Fig. 70 nach HeIDENHAIN.

Fig. 47-50. Hemogregarina sporophilae; Schizogonie.

Fig. 51-58. Hemogregarina sporophilae; Junge und erwachsene Parasiten im Innern der Leukozyten.

Fig. 59. Junge Formen von Schizogonie herrührend.

Fig. 60-63. Hemogregarina tanagrae.

Fig. $64-7 x$, 73 \& 74. Hemogregarina sicalidis.

Fig. 72. Hemogregarina sicalidis; Schizogonie.

Fig. 74, 75,

77 \& 79. Hemogregarina brachyspisae.

Fig. 76. Hemogregarina brachyspisae; Schizogonieform.

\section{BIBLIOGRAFIA.}

ADIr

LAVERAN

Miller W.

Porter, A.

Prowazk, S. v.
1909 Note on a parasite in the sparrow.

Indian Medical Journal, p. I76.

1900 Au sujet de l'hématozoaire endoglobulaire de Padda Orisivora.

Compt. Rend. d. 1. Soc. de Biologie I3-I-oo.

1908 Hepatozoon perniciosum n. g. n. sp.; a hamogregarine patho- to genic for white rats.

Bull. n. 46. Hyg. Lab. U.S.A. Public Health and Mar.

San Dept.

1909 The Leucocytozoa.

Science Progress n. 14 .

1907 Untersuchungen über Haemogregarinen.

Arbeit. a. d. Ksl. Gesundheitsamte. Vol. 26, p. $3^{6 .}$ 

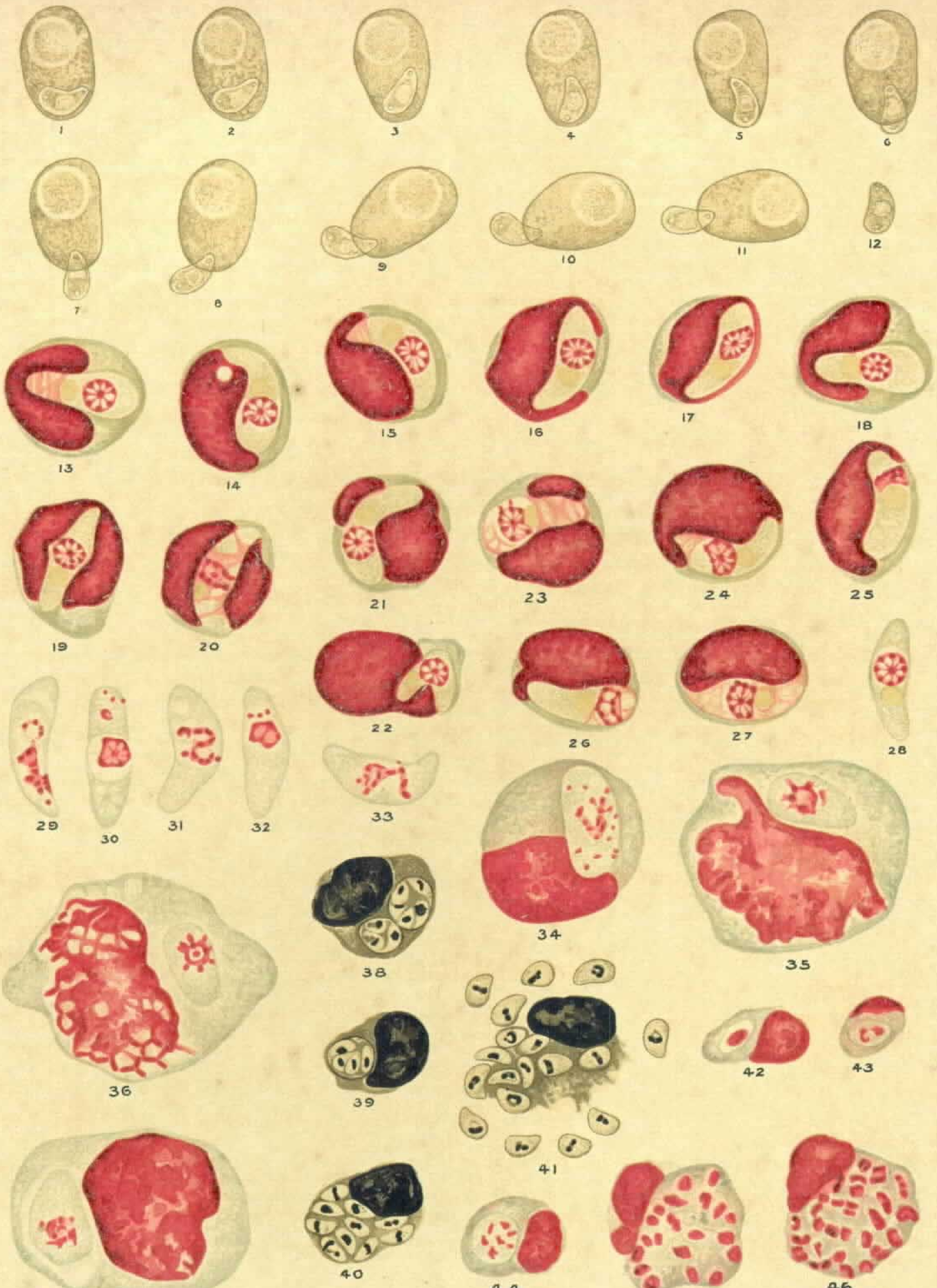

37

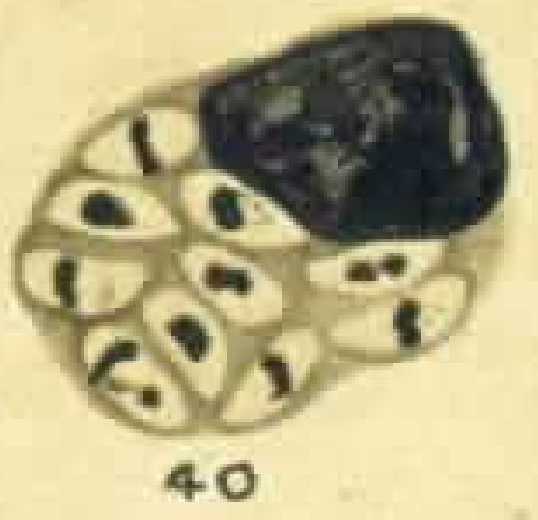

$$
\text { - (b) }
$$
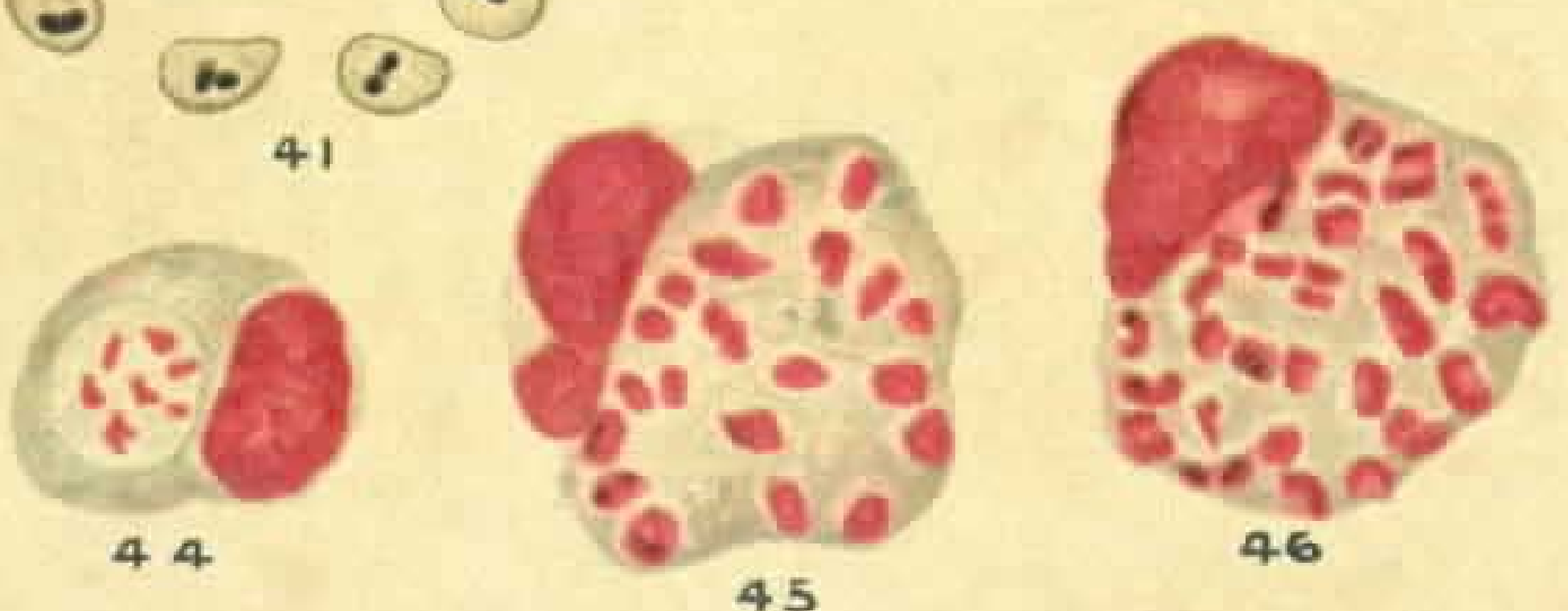

CastroSilva del. 

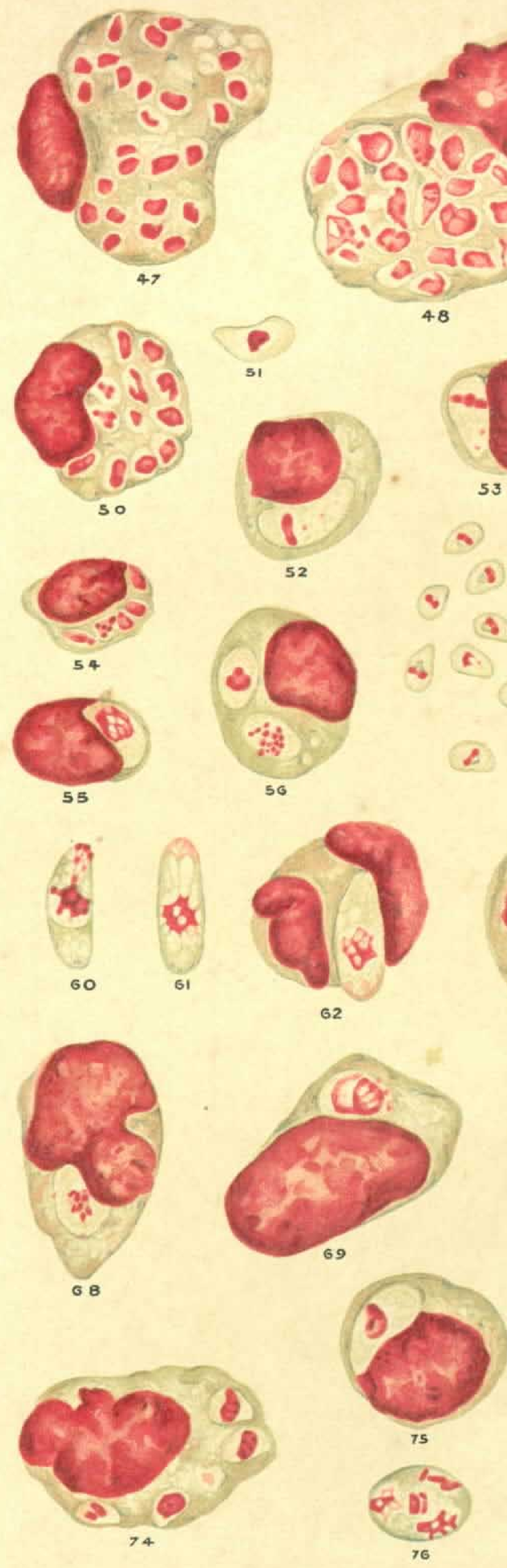
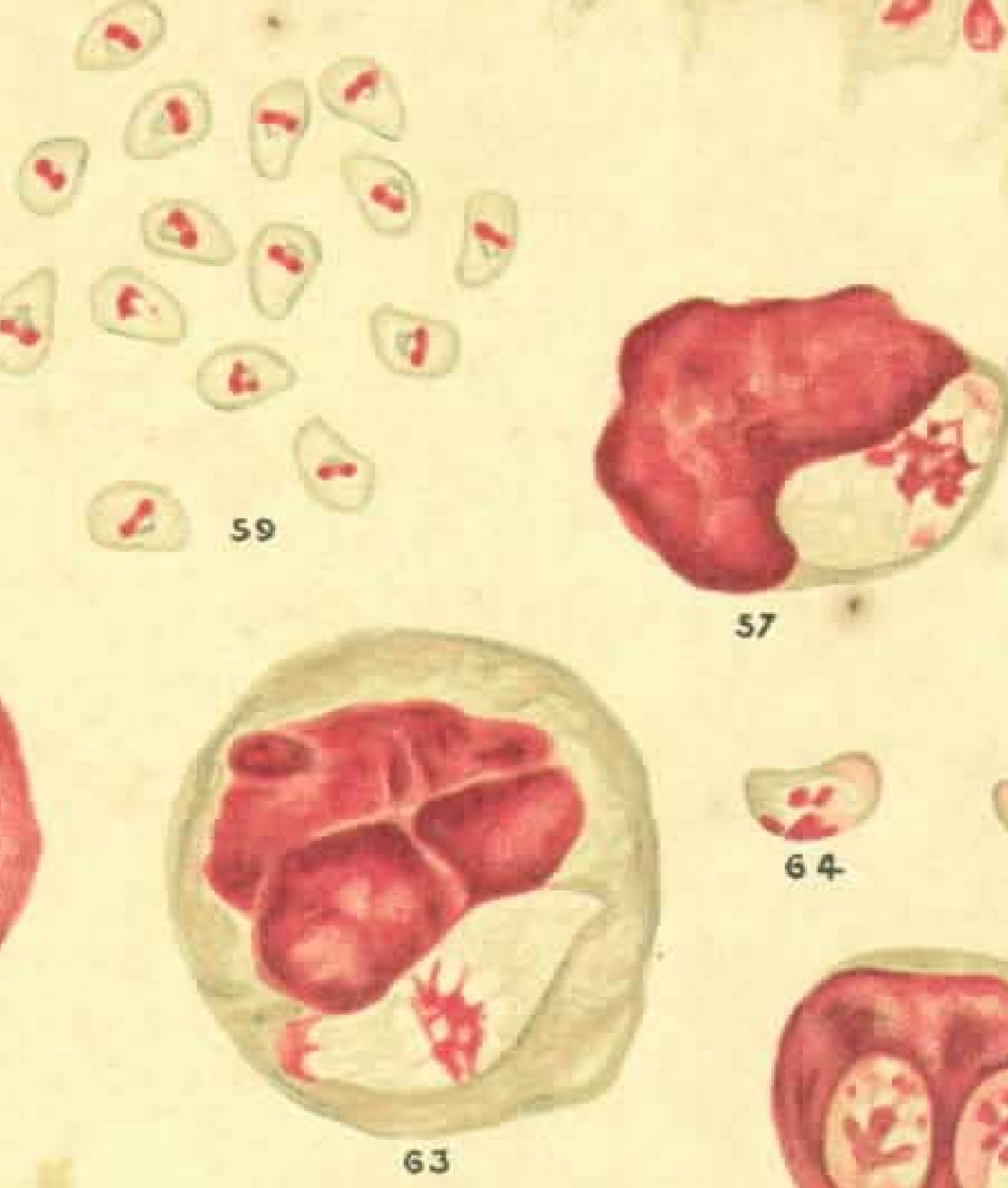
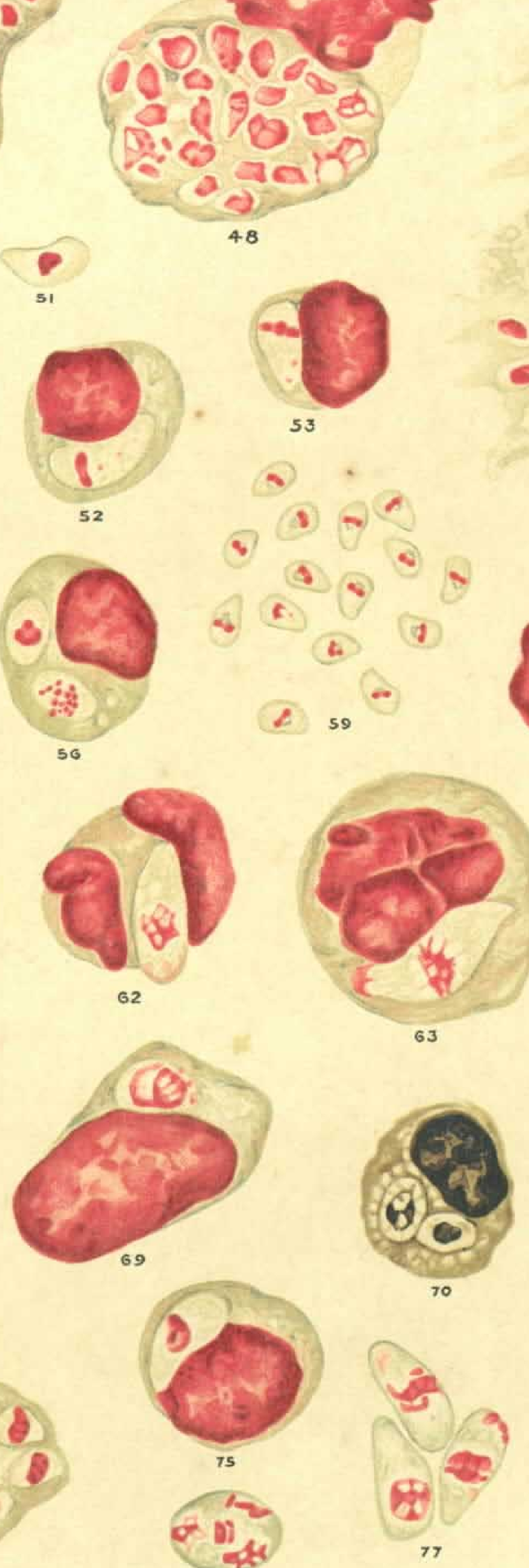
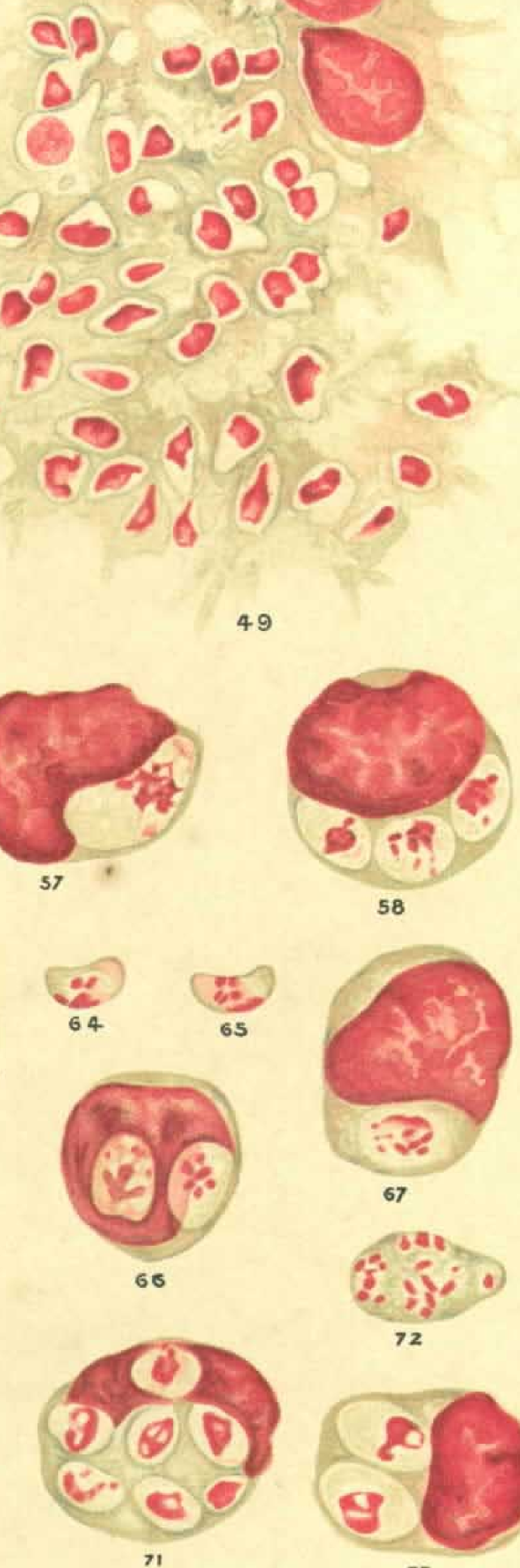

$0^{\circ}>\rho^{\circ}$

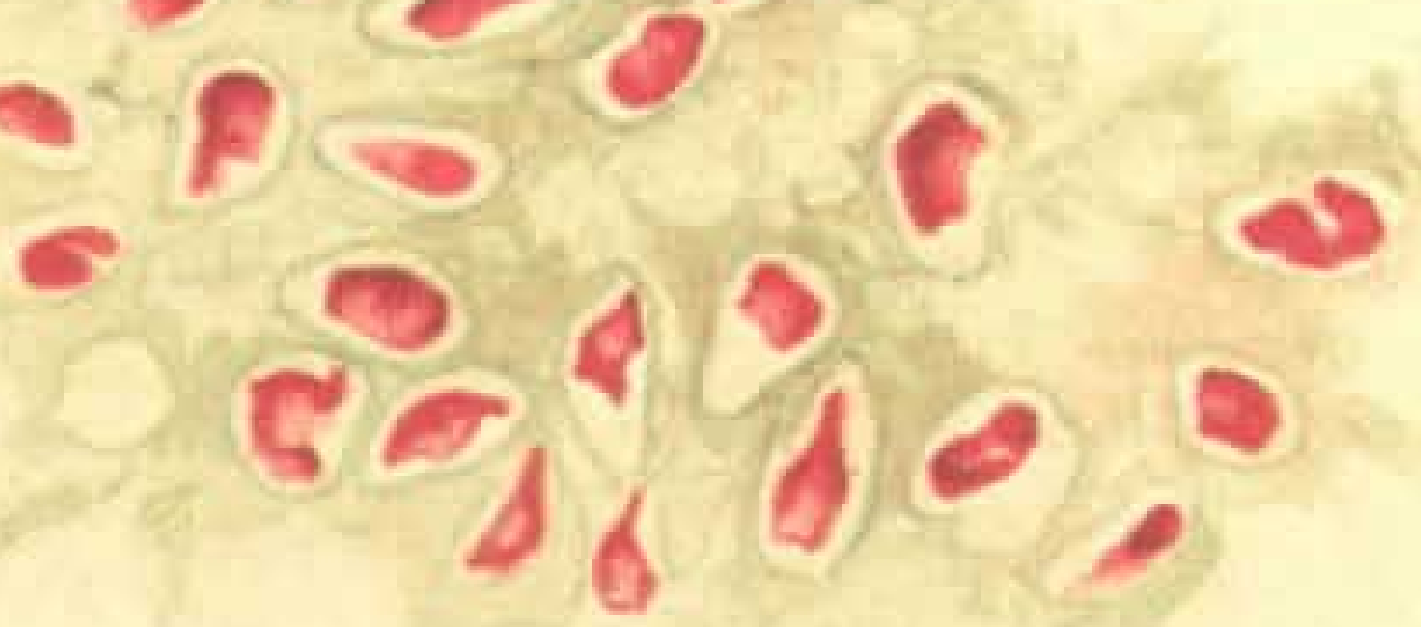

49
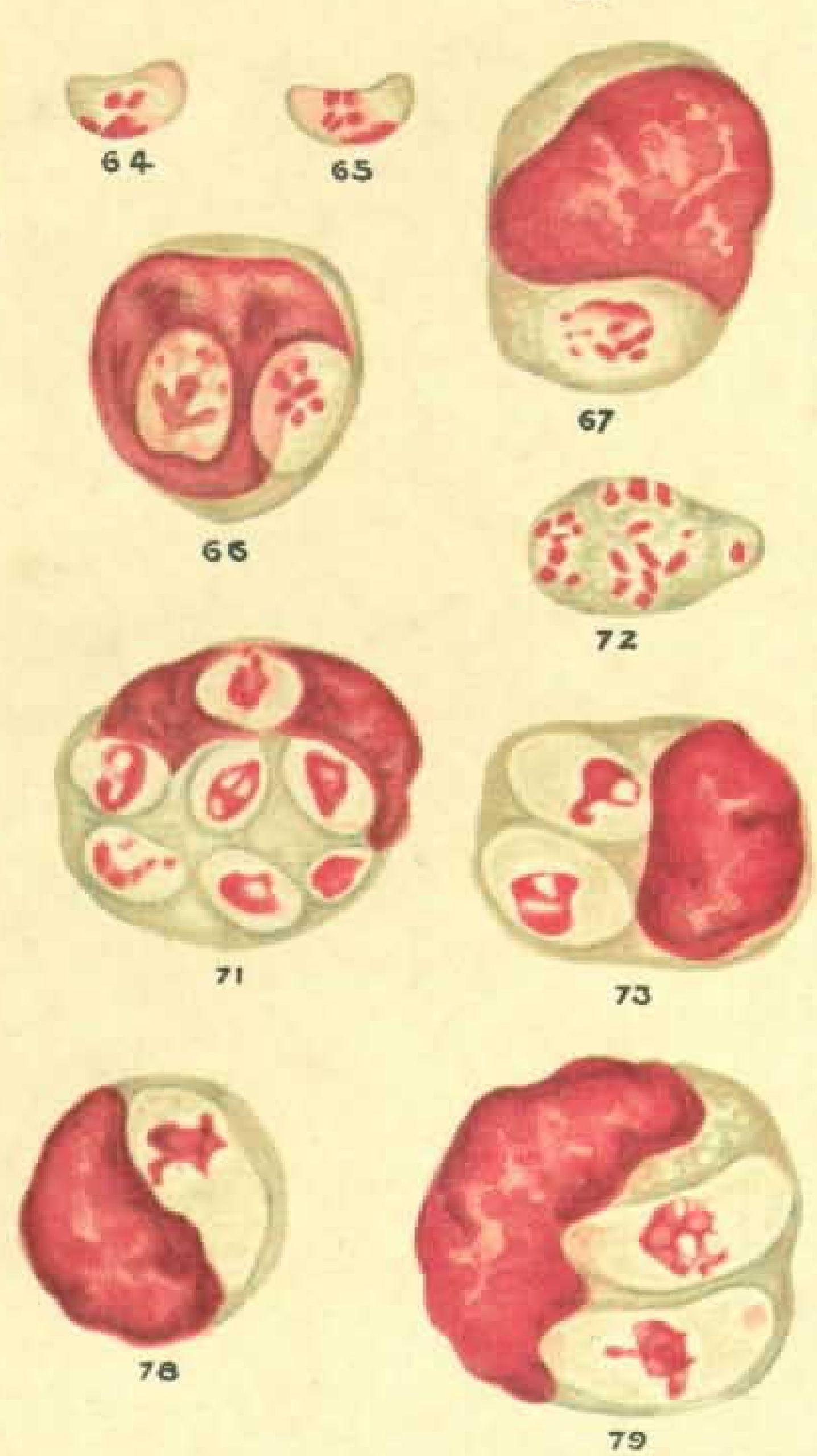

Castrosilva.del. 